\title{
Editorial
}

\section{Perinatal mental health service development across the UK - many achievements, growing challenges}

\author{
Roch Cantwell \\ Perinatal Mental Health Scotland, National Services Scotland, Gyle Square, 1 South Gyle Cresecent, Edinburgh EH12 9EB, UK
}

\begin{abstract}
Perinatal mental health service development had early beginnings in the UK, and there has been rapid expansion over the past decade. As individual nations address need in their own jurisdictions, differences in service models and priorities have emerged. Innovative opportunities for addressing maternal and infant mental health needs must be balanced against the demands brought about by societal change and the COVID-19 pandemic. With such significant change, there is a unique opportunity for services and service planners to share good practice and learn from others' successes and challenges.
\end{abstract}

Keywords: Mental health services; maternal health; mental disorders

(Received 12 December 2021; accepted 16 December 2021)

In 2014, the Royal College of Psychiatrists' Centre for Quality Improvement (CCQI) launched the community arm of its Perinatal Quality Network with the purpose of setting common standards and an accreditation process for specialist services throughout the UK. There were eight founder services. That number now stands at 55 and rising. This huge expansion in specialist provision hides a growing divergence in how services are organised across the four devolved national health services of the UK. Such divergence risks losing the core ethos of what makes a good perinatal mental health service but also opens possibilities for new innovations in care.

The UK has a long history of perinatal mental health service provision. In 1988, Oates described the provision of 'an integrated community-orientated service for severe postnatal mental illness', introducing the principle of comprehensive services across the inpatient and community spectrum (Oates 1988). That model gradually spread throughout England and, more recently, Scotland, Wales and Northern Ireland, largely driven by clinicians with a desire to see better care. However, two specific factors brought about a step change in the pace of development. These were first, lessons on maternal suicide from the Confidential Enquiries into Maternal Deaths and second, the voices of women with lived experience, heard directly or through campaigning organisations.

\section{Drivers for service development}

The UK Confidential Enquiries into Maternal Deaths (CEMD) have reported on all deaths in relation to childbearing since the

Address for correspondence: Roch Cantwell, National Services Division, NHS National Services Scotland, Gyle Square, I South Gyle Crescent, Edinburgh EH12 9EB, UK. (Email: roch.cantwell2@nhs.scot)

Cite this article: Cantwell R. Perinatal mental health service development across the UK - many achievements, growing challenges. Irish Journal of Psychological Medicine https://doi.org/10.1017/ipm.2022.1 1950s. From the early 1990s, deaths due to psychiatric causes have been examined in detail. The Enquiries' methodology focuses on individual case reviews and makes recommendations to improve patient care and service provision (Oates 2003). More recently, Ireland has become a partner in the Enquiries.

In 2001, CEMD recommended that all women should have access to a specialist perinatal mental health team (Oates 2001). By 2015, that was refined to suggest that teams should have the capacity to case manage women with significant perinatal mental illness (Cantwell et al. 2015). But it is often the clinical care lessons from the Enquiries that have been the primary driver of change, including the recognition that existing general services were not easily adapted to provide care that included preventative interventions for women at high risk and close working relationships with maternity, health visiting and child welfare professionals. The Enquiries' lessons have been widely disseminated and helped to create an expectation of service provision across the UK.

There has always been a vocal, well-informed lived experience voice within the perinatal 'family', contributing to service development. In 2011, the establishment of the Maternal Mental Health Alliance (MMHA) brought a range of third sector, professional and service-user organisations together to campaign for service improvement across the UK. MMHA embarked on a number of campaigns to highlight women's voices about their care experience and to bring the 'postcode lottery' of service provision to the attention of health service providers and the public.

Both of these developments led to a recognition of the need for investment in service development at governmental level. In 2016, the UK government announced a programme of investment in perinatal mental health services over the subsequent five years in England. Similar financial commitments were made by the devolved administrations in Wales (2016), Scotland (2019) and Northern Ireland (2021). 


\section{Agreed service models}

An established model for inpatient and community perinatal mental health services was described in the Royal College of Psychiatrists' 2015 report on the provision of services for childbearing women, updated in 2021 (RCPsych 2021). Many of its recommendations echo the accreditation standards of the CCQI Perinatal Quality Network, thus ensuring a high degree of consistency of service design, reflected in what was recommended in individual nations' documents guiding service development. In addition, three of the four nations now have MBU facilities and discussions are underway in Northern Ireland about how best to deliver inpatient care.

As each nation's services evolve, differing emphasis has led to subtle alterations in the model of care. England has proposed the expansion of community specialist provision for up to two years after delivery, the provision of four new MBUs in areas previously sparsely covered, the development of maternity psychology services and improved access to help for fathers (NHSE 2019). In Scotland, different models of community provision have been recommended to take into account rural and low birth number populations, the need to increase inpatient provision, the development of maternity psychological therapies services and the integration of parent-infant provision and peer support workers within specialist services (PMHN Scotland 2019). Wales has prioritised the expansion of community services and has developed a new mother and baby unit (National Assembly for Wales 2017).

In Northern Ireland, a review was conducted by the RQIA in 2017, which involved visits to each health and social care (HSC) trust. It recommended adaptations to general adult wards, to allow for children visiting, in the absence of dedicated MBU facilities, the development of a model of specialist community provision in each HSC trust and exploration of the need for MBU provision. More recently, there has been specific investment in community services with multidisciplinary teams recommended to include psychiatry, clinical psychology, mental health nursing, nursery nursing, occupational therapy, social work, specialist midwifery, specialist health visiting, special interest obstetrician, parent-infant therapy and peer support.

\section{Innovations in service design and delivery}

\section{Peer support workers}

Alongside the movement for true co-production between professionals and patients, there has been a steady expansion of peer support, provided by people with lived experience, within specialist inpatient and community settings. Peer support workers are now recommended as core members of MBU and community teams and are included in CCQI standards, and guidance provided about core roles (McPin Foundation 2019; PMHN Scotland 2020). There is no single model of peer support provision and approaches may include direct employment by the NHS clinical service or contractual arrangements with third sector organisations, but it is important that peer support workers are appropriately remunerated for their expertise. Challenges remain to ensure that peer workers have adequate support and supervision in a mental health setting, that they are truly seen as equal members of the team and that their own mental health needs are respected.

\section{Parent-infant therapists}

While the importance of sustaining the mother-infant relationship and promoting infant development has long been recognised as a core function of perinatal mental health services, in practice, much of specialist team training and function has focussed on the management of maternal mental disorder. The development of a small number of exemplar services, coupled with the wider recognition of the importance of the early years' environment, led to a re-evaluation of the need for greater inclusion of parent-infant interventions in specialist services. England, Northern Ireland and Scotland now include parent-infant therapists as recommended core members of MBU and community teams.

\section{Maternity psychological services}

Providing liaison to maternity units has always been a core function of specialist services, but it has tended to focus on women with, or at risk of, the range of disorders typically presenting to secondary care mental health services. There has been a gap in provision for women experiencing more common mental health difficulties in a maternity setting. These would include those with birth trauma, pregnancy or neonatal loss and parents adjusting to significant obstetric or neonatal complications.

In response to this need, Scotland recommended the development of Maternity and Neonatal Psychological Interventions (MNPI) Services, based in larger maternity units, staffed by psychologists and perinatal mental health midwives (PMHN Scotland 2019). A similar initiative in England, termed Maternal Mental Health Services, is in the process of piloting models of care (NHSE 2019).

\section{Continuity up to 2 years}

NHS England's Long Term Plan has proposed that community perinatal mental health services should extend their remit to the end of the second postnatal year (NHSE 2019). It remains unclear at present whether this change in criteria would be for all new referrals to services or only for those already in the care of or previously known to the specialist team.

\section{Challenges for service delivery}

\section{Remote and rural provision}

There is a particular challenge for areas with low or very dispersed birth population numbers in ensuring they have access to specialist care. In dispersed populations, travelling time can be a significant barrier to face to face contact. The pandemic has provided an unexpected response in the rapid adoption of digital technologies. While useful, there can be problems in making thorough clinical assessments online, particularly in a perinatal setting. Guidance has been developed around these particular challenges (TEC Scotland 2020).

For very low birth, remote and rural areas, guidance has been provided in the recent Royal College of Psychiatrists' report on service provision (RCPsych 2021) which suggested that service models should include enhanced skills for mental health professionals in generic teams accompanied by regional links to specialist perinatal mental health teams who can provide additional supervision and support in clinical decision making. Remote, rural and island populations have also been addressed in the model of service provision underway in Scotland (PMHN Scotland 2019). Three models of community specialist team are described - stand-alone for large population areas, dispersed (a small core team with additional dispersed staff, with dedication time, embedded in existing community mental health teams) for low population areas and regional (additional training for generic staff with links specialist teams in 
larger centres) for very low birth and island areas. Implementation is ongoing with evaluation built into the national programme.

\section{Widening existing access in relation to sex and gender}

Families may be constituted in many different ways. Services are increasingly likely to be challenged to provide a clinical response to parents and their infants who have not been included in mother and baby care previously. These include not only fathers who are the sole primary carer of their infant or who are in same sex relationships, and who develop significant mental illness, but also transsexual men who are pregnant or postnatal.

Widening access in this regard increases demand on services and, for MBUs in particular, challenges the traditional model of delivering care. It also requires reflection on our use of inclusive language to describe the services we provide.

\section{Services for substance misuse}

The needs of pregnant and postnatal women with substance misuse, and their infants, have often been overlooked in the development of perinatal mental health provision. Women with substance misuse are at greater risk of suicide and deaths by accidental overdose in the perinatal period, and vulnerability increases in the context of child protection proceedings (Oates \& Cantwell 2011). Their infants are more likely to be removed into care and, for alcohol misuse in particular, to experience developmental difficulties. Yet, there is no one accepted model of care to address need and limited models of preventative interventions.

While traditional perinatal mental health care might not be adaptable to this group, we have a clear duty to assist in the development of suitable care. There are some signs that this conversation has begun.

\section{Maintaining existing access}

With rapid expansion comes the risk that the core roles of perinatal mental health services are lost. In the face of increasing demand, there may be a tendency to narrow the definition of perinatal mental illness to those conditions that show a clear association with pregnancy or the postnatal state (e.g., postpartum psychosis, newly arising depressive disorders). This fundamentally misunderstands the critical role of specialist services in assessment and treatment given the modifying effects of pregnancy and parenting on the course of pre-existing mental illness, the need for a distinctive response in terms of liaison with maternity, primary care and child welfare colleagues, pharmacological management in pregnancy and breastfeeding and the need for interventions tailored at addressing the parent-infant relationship and infant development.

As the landscape of other services alters, there is also a need for close liaison between teams who may have joint care of women and their infants at this time, such as early intervention in psychosis services, home treatment teams and mental health assessment centres. Perinatal mental health services need to make the case for continued involvement, in many circumstances as the lead care coordinating service.

\section{COVID-19}

The COVID-19 pandemic has increased the demand for mental health care, and the perinatal period is a time of particular vulnerability (Papworth et al. 2021). Women have been isolated from the family and social supports usually expected during pregnancy and early parenting. Infants have had less opportunity to engage with extended family and peers. Maternity and mental health care have been altered, with reduced face to face contact and almost complete cessation of group interventions.

Paradoxically, the increased use of telemedicine has improved access for some women and families but comes at a price of restricting ability to make comprehensive assessments of women at high risk or in vulnerable social circumstances. Guidance is available to help services recognise and overcome these limitations in clinical encounters (TEC Scotland 2020). As we come out of the acute phase of the pandemic, we need to not only hold on to the changes in ways of working that have benefitted our patients but also recognise that demand, and the pattern of that demand may remain altered from what we had encountered before. Services should be responsive to change, but must prioritise clinical need rather than, from an often very vulnerable position, patients are forced to adapt to our unchallenged models of care.

Conflicts of interest. Authors have no conflicts of interest to disclose.

Ethical standards. The authors assert that all procedures contributing to this work comply with the ethical standards of the relevant national and institutional committee on human experimentation with the Helsinki Declaration of 1975, as revised in 2008 .

Financial support. This research received no specific grant from any funding agency, commercial or not-for-profit sectors.

\section{References}

Cantwell R, Knight M, Oates M, Shakespeare J on behalf of the MBRRACEUK mental health chapter writing group (2015). Lessons on maternal mental health. In Saving Lives, Improving Mothers' Care - Surveillance of maternal deaths in the UK 2011-13 and lessons learned to inform maternity care from the UK and Ireland Confidential Enquiries into Maternal Deaths and Morbidity 2009-13 (ed. Knight M, Tuffnel D, Kenyon S, Shakespeare J, Gray R, Kyrinczuk JJ, on behalf of MBRRACE-UK), pp. 22-41. National Perinatal Epidemiology Unit, University of Oxford: Oxford.

McPin Foundation (2019). Peer support principles for maternal mental health. (https://mcpin.org/wp-content/uploads/2019/12/Peer-Support-Principlesfor-perinatal-mental-Health-2019-FINAL.pdf). Accessed 7 June 2021

National Assembly for Wales (2017) Perinatal mental health in Wales: a report of the Childrens, Young People and Education Committee. (https://senedd. wales/laid\%20documents/cr-ld11234/cr-ld11234-e.pdf). Accessed 7 June 2021.

NHS England (2019) The NHS Long Term Plan. (https://www.longtermplan. nhs.uk/wp-content/uploads/2019/08/nhs-long-term-plan-version-1.2.pdf) Accessed 7 June 2021.

Oates M (1988). The development of an integrated community service for severe postnatal mental illness. In Motherhood and Mental Illness 2: Causes and Consequences (ed. Kumar S, Brockington IF), pp. 133-158. London: Wright.

Oates MR (2001). Deaths from psychiatric causes. In Why Mothers Die 19971999: The Fifth Report of the Confidential Enquiries into Maternal Deaths in the United Kingdom (ed. Botting BJ, Lewis G), Royal College of Obstetricians and Gynaecologists: London.

Oates M (2003). Suicide: the leading cause of maternal death. British Journal of Psychiatry 183, 279-281.

Oates M, Cantwell R (2011). Deaths due to psychiatric causes. In Saving Mothers' Lives: Reviewing maternal deaths to make motherhood safer 2006-2008: The Eighth Report of the Confidential Enquiries into Maternal Deaths in the United Kingdom (ed. Lewis G), pp. 132-142.

Papworth R, Harris A, Durcan G, Wilton J, Sinclair S (2021) Maternal mental health during a pandemic: a rapid evidence review of Covid-19's impact. (https://maternalmentalhealthalliance.org/wp-content/uploads/ CentreforMH_MaternalMHPandemic_FullReport.pdf) Accessed 7 June 2021. 
PMHN Scotland (2019) Delivering Effective Services: needs assessment and service recommendations for specialist and universal perinatal mental health services. (https://www.gov.scot/publications/delivering-effective-servicesneeds-assessment-service-recommendations-specialist-universal-perinatalmental-health-services/). Accessed 7 June 2021.

PMHN Scotland (2020) Specialist perinatal mental health professionals: role definitions and support structures - perinatal mental health peer support worker. (https://www.pmhn.scot.nhs.uk/wp-content/uploads/2020/11/ Role-definitions_peer-support-worker_Final.pdf) Accessed 7 June 2021.
RCPsych (2021). College Report 232. Perinatal Mental Health Services: recommendations for the provision of services for childbearing women. RCPsych: London.

RQIA (2017) Review of perinatal mental health services in Northern Ireland. (https://www.rqia.org.uk/RQIA/files/28/28f4ee85-a5e9-4004-b922525bc41ae56d.pdf) Accessed 7 June 2021.

TEC Scotland (2020) Coronavirus resilience planning: telepsychiatry quick reference guide 4 - telepsychiatry in perinatal mental health settings. (https://tec. scot/nearme/clinical-specialty-guidance/) Accessed 7 June 2021. 